\title{
Measuring the bias of incorrect application of feature selection when using cross-validation in radiomics
}

\author{
Aydin Demircioğlu* (1)
}

\begin{abstract}
Background: Many studies in radiomics are using feature selection methods to identify the most predictive features. At the same time, they employ cross-validation to estimate the performance of the developed models. However, if the feature selection is performed before the cross-validation, data leakage can occur, and the results can be biased. To measure the extent of this bias, we collected ten publicly available radiomics datasets and conducted two experiments. First, the models were developed by incorrectly applying the feature selection prior to cross-validation. Then, the same experiment was conducted by applying feature selection correctly within cross-validation to each fold. The resulting models were then evaluated against each other in terms of AUC-ROC, AUC-F1, and Accuracy.
\end{abstract}

Results: Applying the feature selection incorrectly prior to the cross-validation showed a bias of up to 0.15 in AUCROC, 0.29 in AUC-F1, and 0.17 in Accuracy.

Conclusions: Incorrect application of feature selection and cross-validation can lead to highly biased results for radiomic datasets.

Keywords: Radiomics, Feature selection, Cross-validation, Bias, Machine learning

\section{Key points}

- Incorrectly applying feature selection on the whole dataset before cross-validation can cause a large positive bias.

- Datasets with higher dimensionality, i.e., more features per sample, are more prone to positive bias.

\section{Background}

Radiomics is a method to extract and analyze highdimensional quantitative features from radiological, non-invasive imaging data to enable predictive decision support [1]. The basic assumption of radiomics is that

*Correspondence: aydin.demircioglu@uk-essen.de Institute of Diagnostic and Interventional Radiology and Neuroradiology, University Hospital Essen, Hufelandstr. 55, 45147 Essen, Germany these features correspond to imaging biomarkers that contain characteristic information about diseases. Radiomics potentially allows for patient-centric diagnosis [2] and has been employed for many types of tumors [3-6].

Since it is not known beforehand which feature will be important for the particular outcome considered, radiomics extracts far more features than necessary. Many of these are therefore potentially irrelevant and redundant [7-9]. Thus, various feature selection methods are employed to reduce the features to the most predictive and robust ones, although it is well known that these methods are challenging and can be misleading [10].

Another problem with radiomics is the rather small sample sizes. There are several reasons for this, for example, if the pathology under consideration is rare or suitable data is not readily available. In addition, radiomics often needs segmentations of the pathology, which cannot be performed manually if the sample sizes go into the thousands. Also, access to external 
data is often restricted because of privacy issues. Therefore, radiomic datasets often comprise only a few hundred samples, which is critical from a statistical viewpoint [11].

Together with the multitude of extracted features, this leads to high-dimensional datasets, i.e. they have fewer samples than features. Since the analysis of such datasets is complex, guidelines and standards were introduced to ensure the validity of radiomics studies [12-15].

A key problem when modeling is overfitting, which occurs when a model learns the noise and peculiarities of a given training dataset rather than the underlying patterns, and therefore does not generalize to new data. Overfitting problems can be identified by using validation data that is not used during training. Since explicit validation data is rarely available, validation schemes are used, where part of the training data is set apart and only used to obtain an unbiased estimate of the performance of the model. Often, cross-validation $(\mathrm{CV})$ is employed, where the data is split into several folds and then used in turn to train and to validate the model.

When cross-validation is employed, clearly all modeling must be applied only to the training folds, else data leakage would occur, which describes the fact that the validation data was already used and estimations could potentially be biased. This is especially true for the feature selection, which is a fundamental part of the radiomics pipeline. Since applying the feature selection before the cross-validation on all data would lead to data leakage, feature selection must be part of the cross-validation for the resulting model to be unbiased and to generalize to new data.

Unfortunately, sometimes illustrations of the radiomics pipeline are simplified and make the impression that feature selection is a preprocessing step before modeling applied to the whole data and that cross-validation is only part of the model selection $[3,12,16]$. Similarly, it is not always clear whether studies that use cross-validation but not an explicit validation set have applied feature selection incorrectly or whether it is just misleadingly described [17-29]. In fact, only a few studies describe their methodology in full [6].

To understand how far incorrect application of feature selection before cross-validation introduces a bias to the analysis, it is important to measure the extent of the difference. Therefore, in this study we utilized 10 radiomics datasets, 7 feature selection methods as well as 7 classifiers and study via a tenfold cross-validation in how far an incorrect order of feature selection and cross-validation has an impact on the estimated performance.

\section{Methods}

All data used in this study were previously published; therefore ethical approval was waived by the local Ethics Committee (Ethik-Kommission, Medizinische Fakultät der Universität Duisburg-Essen, Germany). Methods and procedures were performed in accordance with the relevant guidelines and regulations.

\section{Data collectives}

For the reproducibility of our study, publicly available datasets are paramount. We therefore scanned the open-access journal "PLOS One" using the search key "radiomics" for papers that share their data publicly. Ten such studies have been identified and the data has been included into this study (Table 1). For reproducibility, all datasets were placed in a public repository (https:/github.com/aydindemircioglu/radCV). All datasets were high-dimensional with two exceptions: Carvalho2018, which is the only low-dimensional dataset, and Song2020, which is almost low-dimensional. Here, we call a dataset high-dimensional if it has fewer samples than features, and low-dimensional otherwise.

For each dataset, all available data, even if it was previously split into training and validation sets, was merged. This was performed to minimize any effect of non-identically distributed data on the prediction, which would potentially introduce a different bias. In the same spirit, all clinical features were removed, as the focus was only on the highly redundant and correlated radiomics features. More details can be found in Additional file 1.

\section{Cross-validation}

Since some imbalance in the outcome was seen in a few datasets, stratified tenfold cross-validation was employed, i.e. while splitting of each dataset into 10 evenly sized folds it was made sure that the outcome balance in each fold was similar to the balance of the whole dataset. Cross-validation scores were computed by micro-averaging, i.e. first predictions from the 10 folds were pooled and then relevant metrics like AUCROC were computed on the pooled data.

\section{Preprocessing}

An important step is the preprocessing of data whose main task is to harmonize the data. To avoid positive bias, especially in the presence of outliers, preprocessing must also take place inside the cross-validation. Despite this, in this study preprocessing was applied before the cross-validation to the whole dataset. This was done because application of preprocessing steps inside the cross-validation might interfere and occlude 
Table 1 Overview of the datasets

\begin{tabular}{|c|c|c|c|c|c|c|c|}
\hline Dataset & $N$ & $d$ & $\begin{array}{l}\text { Dimensionality } \\
\text { (\#Samples/\#Features) }\end{array}$ & $\begin{array}{l}\text { Outcome } \\
\text { balance } \\
(\%)\end{array}$ & Modality & Tumor type & DOI \\
\hline Carvalho2018 [30] & 262 & 117 & 2.22 & 59 & FDG-PET & NSCLC & $\begin{array}{l}\text { https://doi.org/10.1371/journal.pone. } \\
0192859\end{array}$ \\
\hline Hosny2018A (HarvardRT) [31] & 293 & 1004 & 0.29 & 54 & $\mathrm{CT}$ & NSCLC & $\begin{array}{l}\text { https://doi.org/10.1371/journal.pmed. } \\
1002711\end{array}$ \\
\hline Hosny2018B (Maastro) [31] & 211 & 1004 & 0.21 & 28 & $\mathrm{CT}$ & NSCLC & $\begin{array}{l}\text { https://doi.org/10.1371/journal.pmed. } \\
1002711\end{array}$ \\
\hline Hosny2018C (Moffitt) [31] & 183 & 1004 & 0.18 & 73 & $\mathrm{CT}$ & NSCLC & $\begin{array}{l}\text { https://doi.org/10.1371/journal.pmed. } \\
1002711\end{array}$ \\
\hline Ramella2018 [32] & 91 & 242 & 0.37 & 55 & $\mathrm{CT}$ & $\mathrm{NSCLC}$ & $\begin{array}{l}\text { https://doi.org/10.1371/journal.pone. } \\
0207455\end{array}$ \\
\hline Toivonen2019 [33] & 100 & 7105 & 0.01 & 60 & MRI & Prostate Cancer & $\begin{array}{l}\text { https://doi.org/10.1371/journal.pone. } \\
0217702\end{array}$ \\
\hline Keek2020 [34] & 273 & 1322 & 0.21 & 40 & $\mathrm{CT}$ & HNSCC & $\begin{array}{l}\text { https://doi.org/10.1371/journal.pone. } \\
0232639\end{array}$ \\
\hline Li2020 [35] & 51 & 396 & 0.13 & 63 & MRI & Glioma & $\begin{array}{l}\text { https://doi.org/10.1371/journal.pone. } \\
0227703\end{array}$ \\
\hline Park2020 [36] & 768 & 940 & 0.82 & 24 & US & Thyroid Cancer & $\begin{array}{l}\text { https://doi.org/10.1371/journal.pone. } \\
0227315\end{array}$ \\
\hline Song2020 [37] & 260 & 264 & 0.98 & 49 & $\mathrm{MR}$ & Prostate Cancer & $\begin{array}{l}\text { https://doi.org/10.1371/journal.pone. } \\
0237587\end{array}$ \\
\hline
\end{tabular}

Overview of all radiomics datasets used. Only publicly available datasets were included to allow for easy reproducibility. $N$ denotes the sample size, while $d$ denotes the number of features (corresponding to the dimension of the data). The outcome balance measures the number of events in the outcome. DOI denotes the identifier of the publication corresponding to the dataset

the bias arising from the incorrect application of feature-selection before the cross-validation. Since this effect was the main focus, preprocessing was performed upfront on the whole dataset.

Two preprocessing steps were applied: Imputation and normalization. The imputation was necessary as a few datasets had missing values. Such missing values can occur when computing large numbers of radiomics features because of numerical problems. The number of missing values was well below $1 \%$ for each feature and dataset. Imputation was performed by using columnwise means. Normalization using z-scores was applied afterwards.

\section{Feature selection}

The goal of a feature selection is to remove redundant and irrelevant features. Redundant features are those which can equally well be expressed by other features, while irrelevant features are those which do not contribute to the performance of the model. While redundancy only depends on the data itself and not on the outcome, relevancy is only defined in relation to the outcome. Since the outcome is used in a very central way, removing irrelevant features can lead to a high positive bias if applied incorrectly.

The following 7 feature selection methods were used during modeling: LASSO, $\mathrm{t}$-Score, f-Score, MRMRe
(Minimum Redundancy, Maximum Relevance ensemble), ReliefF, MIM (Mutual Information Maximization) and SVM-RFE (Support Vector Machines-Recursive Feature Elimination). All these methods are filtering methods, i.e., they were applied before classification. Each method yielded a scoring on the features, based on which the best features were then selected. The number of selected features was chosen among $1,2,4$, 8,16 and 32 . More information on the feature selection methods can be found in Additional file 1 .

\section{Classifiers}

Since the classifier is the 'heart' of radiomics, its choice is very important. Six classifiers which can be considered state-of-the-art were used: Logistic regression, random forests (RF), support vector machines with radial basis function kernel (RBF-SVM), neural networks (NN), XGBoost and Naive Bayes with Gaussian likelihood function. Each of these classifiers, with the exception of Naive Bayes, has its own hyperparameters, which were chosen from a predefined grid. In addition, a simple, constant classifier was employed that always predicted the majority class. This acts as a baseline which does not depend on the feature selection method. More information on the classifiers can be found in Additional file 1. 

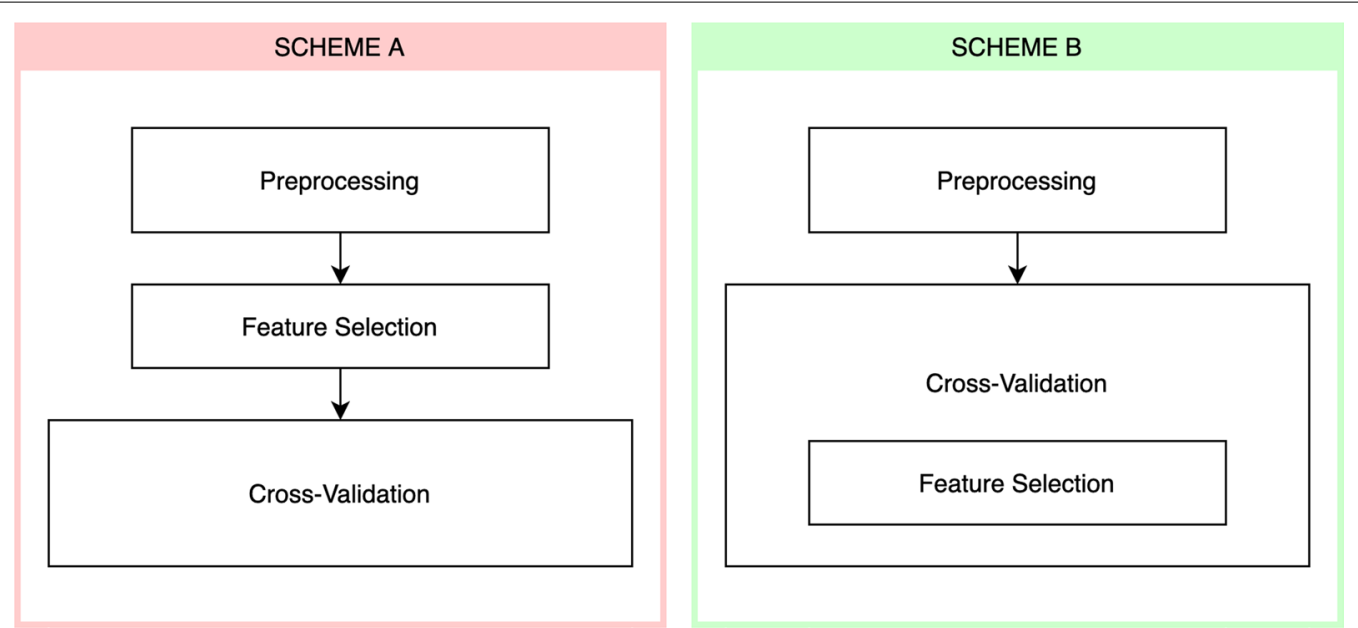

Fig. 1 Illustration of the two training schemes used. In scheme A the feature selection is performed on the whole dataset before the cross-validation, while in scheme B the feature selection is part of the cross-validation, i.e. is applied only to each training fold. Note that preprocessing actually must be part of the cross-validation, but as it would interfere with the bias coming from applying the feature selection incorrectly, it was applied to all data

\section{Training schemes}

Two different training schemes were employed: First, the feature selection was applied to the whole dataset before the cross-validation (Fig. 1, Scheme A). This is incorrect, since the validation fold of the cross-validation was already used for feature selection. Second, the feature selection was correctly applied during the cross-validation separately in each fold (Fig. 1, Scheme B). Because of this, none of the validation folds in the cross-validation were used for feature selection and estimation is therefore not biased by data leakage.

\section{Evaluation}

The performance of each model was measured primarily by AUC-ROC, since in many radiomics analyses AUC$\mathrm{ROC}$ is chosen as the primary metric. More concretely, the model with the highest AUC-ROC using scheme A was selected and compared to the model with the highest AUC-ROC using scheme B. The difference in performance between these two models can be regarded as the bias of incorrectly applying feature selection before cross-validation.

Since higher dimensionality of a dataset, given by the ratio of the number of features to the number of samples, could influence the observed bias, the difference in AUCROC between the two schemes was plotted against the dimensionality. Linear regression was then applied to test if a significant relationship exists between both.

Finally, to understand how far different feature selection methods and classifiers are more prone to bias, we computed the difference in AUC-ROC between the best models for a given feature selection and classifier combination using scheme $\mathrm{A}$ and scheme $\mathrm{B}$. This mimics studies that consider only a single feature selection method and classifier without an extensive search.

In addition to AUC-ROC, the AUC-F1, the area under the precision-recall-curve and the accuracy were also evaluated, but were only considered to be secondary. Other derived metrics, namely sensitivity, specificity, precision and recall and accuracy, were also computed and can be found in Additional file 2.

\section{Statistics}

All descriptive statistics were reported as mean \pm standard deviation. To compare the AUC-ROC, AUC-F1 and accuracy values of two models, bootstrap tests with 2000 repeats were employed. Statistical significance was chosen to be below a p-value of 0.05. Correlation coefficients were computed using Pearson's method. All analyses were conducted with Python 3.6.9 and the scikit learn 0.24 .2 package.

\section{Results}

Altogether, over 50,000 models have been fitted to the 10 datasets. Considering the best model in terms of AUCROC for each dataset, applying the feature selection incorrectly before the cross-validation led always to a positive bias when compared to the correct application of feature selection inside the cross-validation (Table 2). For AUC-ROC, the largest difference was seen for the Hosny2018C dataset $(\triangle \mathrm{AUC}-\mathrm{ROC}=0.149)$ and the smallest one for Song2020 ( $\triangle \mathrm{AUC}-\mathrm{ROC}=0.02)$ (Fig. 2). Based on a bootstrap test for comparing AUC-ROCs of two ROC curves, the difference was highly significant for all 
Table 2 Results of the experiment

\begin{tabular}{|c|c|c|c|c|c|c|c|c|c|}
\hline & AUC-ROC & $\triangle \mathrm{AUC}-\mathrm{ROC}$ & $P$ & AUC-F1 & $\triangle \mathrm{AUC}-\mathrm{F} 1$ & $P$ & Accuracy & $\Delta$ Accuracy & $P$ \\
\hline Carvalho2018 (Scheme A) & 0.687 & 0.041 & 0.33 & 0.733 & 0.011 & 0.791 & 0.634 & -0.004 & 0.913 \\
\hline Carvalho2018 (Scheme B) & 0.646 & & & 0.722 & & & 0.637 & & \\
\hline Hosny2018A (Scheme A) & 0.765 & 0.13 & $<0.001$ & 0.781 & 0.135 & 0.001 & 0.689 & 0.075 & 0.035 \\
\hline Hosny2018A (Scheme B) & 0.636 & & & 0.647 & & & 0.614 & & \\
\hline Hosny2018B (Scheme A) & 0.855 & 0.13 & $<0.001$ & 0.716 & 0.293 & $<0.001$ & 0.791 & 0.09 & 0.001 \\
\hline Hosny2018B (Scheme B) & 0.725 & & & 0.422 & & & 0.701 & & \\
\hline Hosny2018C (Scheme A) & 0.77 & 0.149 & 0.005 & 0.87 & 0.043 & 0.212 & 0.792 & 0.093 & 0.019 \\
\hline Hosny2018C (Scheme B) & 0.621 & & & 0.827 & & & 0.699 & & \\
\hline Ramella2018 (Scheme A) & 0.872 & 0.061 & 0.147 & 0.893 & 0.051 & 0.21 & 0.846 & 0.11 & 0.024 \\
\hline Ramella2018 (Scheme B) & 0.811 & & & 0.842 & & & 0.736 & & \\
\hline Toivonen2019 (Scheme A) & 1 & 0.146 & 0.002 & 1 & 0.038 & 0.015 & 0.98 & 0.17 & $<0.001$ \\
\hline Toivonen2019 (Scheme B) & 0.854 & & & 0.962 & & & 0.81 & & \\
\hline Keek2020 (Scheme A) & 0.765 & 0.086 & 0.005 & 0.714 & 0.14 & 0.001 & 0.725 & 0.07 & 0.018 \\
\hline Keek2020 (Scheme B) & 0.678 & & & 0.575 & & & 0.656 & & \\
\hline Li2020 (Scheme A) & 0.972 & 0.107 & 0.018 & 0.984 & 0.067 & 0.057 & 0.922 & 0.157 & 0.006 \\
\hline Li2020 (Scheme B) & 0.865 & & & 0.917 & & & 0.765 & & \\
\hline Park2020 (Scheme A) & 0.698 & 0.067 & 0.006 & 0.394 & 0.061 & 0.036 & 0.763 & 0.005 & 0.602 \\
\hline Park2020 (Scheme B) & 0.631 & & & 0.333 & & & 0.758 & & \\
\hline Song2020 (Scheme A) & 0.985 & 0.02 & 0.002 & 0.984 & 0.022 & 0.007 & 0.942 & 0.012 & 0.334 \\
\hline Song2020 (Scheme B) & 0.965 & & & 0.962 & & & 0.931 & & \\
\hline
\end{tabular}

AUC-ROC, AUC-F1 and accuracy of the correct and incorrect models for each dataset as well as their differences and significance. The $p$-values were computed using a bootstrap test with the null hypothesis that the difference is zero. Significant $p$-values are marked in bold

datasets $(p<0.005)$ except for Li2020 ( $p=0.018)$, and not significant for Carvalho2018 $(p=0.33)$ and Ramella2018 $(p=0.147)$.

Similarly, in the AUC-F1 as well as the accuracy a positive bias up to 0.293 and 0.17 respectively, could be seen with the only exception of Carvalho2018, which is the only low-dimensional dataset, where a very minor bias in AUC-F1 was seen $(\triangle \mathrm{AUC}-\mathrm{F} 1=0.011)$ and even a slight loss in accuracy $(\triangle$ Accuracy $=-0.004)$.

Plotting the samples per feature number against the observed bias showed a significant negative tendency for AUC-ROC (Pearson correlation $R=-0.72 ; p=0.02$ ), indicating that with fewer samples per feature the likelihood of bias increases (Fig. 3). Similar tendencies could be seen for the F1-score $(R=-0.64, p=0.045)$ and accu$\operatorname{racy}(R=-0.77, p=0.008)$.

To understand how far the bias can be traced back to the feature selection method and the classifier, for each dataset the best AUC-ROC of each combination was considered. Then, the difference to the AUC-ROC of the best model with the same combination, but with incorrectly applied feature selection was measured. The mean of these differences over all dataset was then computed (Fig. 4). The results show that especially LASSO, especially with Logistic regression, RBF-SVM and Neural Networks as classifiers, as well as MIM tend to show high bias, if feature selection is incorrectly applied. On the other hand, it would seem that SVM-RFE is less biased. But even here, e.g. when the SVM-RFE is combined with random forests, on Toivonen2019 a bias of 0.07 in AUCROC can be observed.

The average bias in F1-score was also very high for the LASSO and MIM, and equally lower for the SVM-RFE. Accuracy showed less overall bias, again LASSO shows larger bias than other feature selection methods.

\section{Discussion}

Obtaining reliable models and predictions in radiomics is notoriously difficult because of the high dimensionality of the datasets involved. Accordingly, several guidelines were presented $[12,13]$ and a radiomics score was introduced to safeguard against spurious results and to define best practices [14]. Despite this, it is not evident if all radiomics studies follow best practices.

We have studied how far an incorrectly applied feature selection on the whole dataset before cross-validation leads to a bias because of data leakage. Our results clearly showed that a large positive bias can result from this that can be as high as 0.15 in AUC-ROC, which is the primary metric in many radiomics studies. This underlines the fact that feature selection applied to the whole dataset 

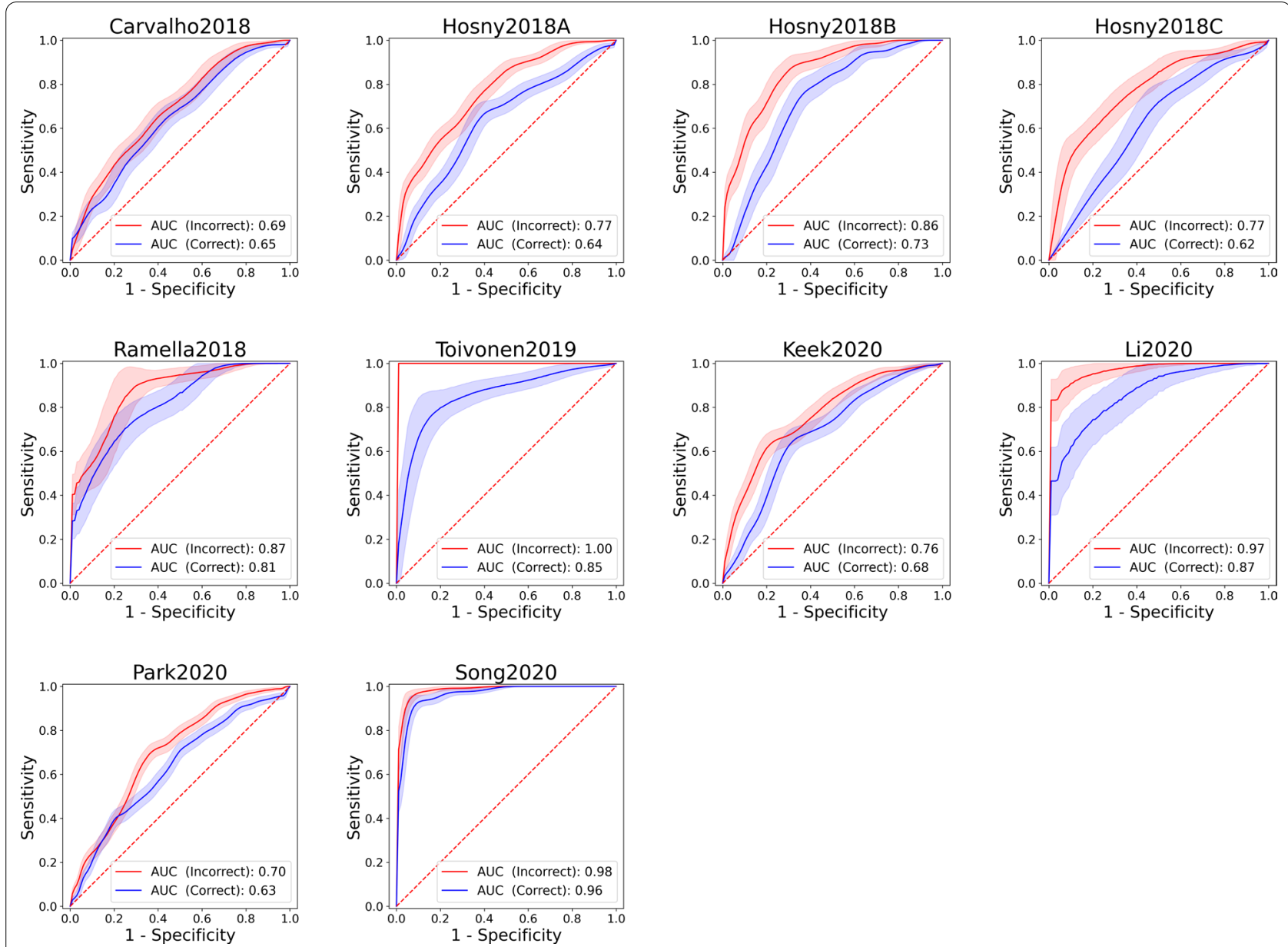

Fig. 2 ROC curves for all datasets. The red and blue curves correspond to application of the feature selection before (Scheme A) and within (Scheme B) the cross-validation

induces a large bias that must be avoided at any cost, if results should be trusted.

While all models showed a positive bias in AUCROC when feature selection was applied incorrectly, the datasets Toivonen2019 and Hosny2018 stand out. On Toivonen2019, the incorrect model yielded an AUC-ROC of 1.0, in stark difference to the analysis by Toivonen et al. which yielded an AUC-ROC of 0.88, comparable to the AUC-ROC of 0.86 we obtained [33]. Similarly, on Hosny2018A and Hosny2018C, predictions were not much different from a random guess (AUC-ROC of 0.62 and 0.64), but became apparently quite usable when using the incorrectly applied feature selection (AUC-ROC of 0.77), in contrast to the study by Hosny et al., since they reported an AUC-ROC of 0.66 [31]. Similar trends could be seen for AUC-F1, although the high bias of 0.293 stemmed from a particularly low AUC-F1 for Scheme B (0.422), which was worse than a random guess. Such performance can happen as we did not select the best model for AUC-F1, but for AUC-ROC. Accuracy also showed a positive bias up to 0.17 , but was non-significant on three datasets.

From the experiment, it seemed that indeed datasets with higher dimensionality, i.e. more features per sample, were more prone to overfitting when feature selection was applied incorrectly. This was to be expected as feature selection is hard and even small data leakage can help to select better features, resulting in a positive bias.

Considering whether certain feature selection methods were more prone to bias than others, it seemed that on average LASSO and MIM showed more positive bias, while SVM-RFE behaved better in this regard. Still, even SVM-RFE showed a positive bias of 0.07 on Toivonen2019. This bias corresponds to an additional 20 patients (of 100) being incorrectly classified as having a prostate cancer with Gleason score $>3+3$, instead of $3+3$ (Biased: $\mathrm{TN}=10, \mathrm{FN}=6, \mathrm{FP}=10, \mathrm{TP}=74$ vs. unbiased: $\mathrm{TN}=13, \mathrm{FN}=26, \mathrm{FP}=7, \mathrm{TP}=54$ ). Thus, the seemingly small average bias cannot be used as a 


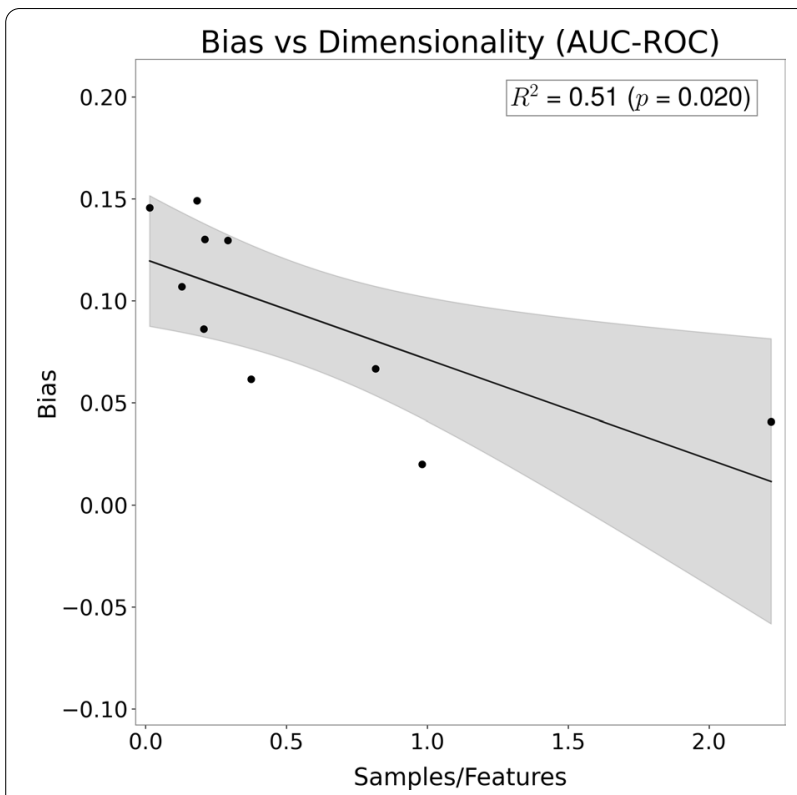

Fig. 3 Scatter plot relating the number of samples per feature against the observed bias in AUC-ROC, when feature selection is applied incorrectly, for each dataset

pretext to circumvent correct application of features selection.

From the plot, it appeared that the feature selection has a much larger impact than the choice of the classifier, which at first sight contradicts the results by Parmar etc. [38]. This arises from the fact that we considered biases, not overall performances. Because the only difference between scheme A and scheme B was whether the feature selection method was able to select better features because of data leakage, this result appears to be reasonable.

Feature selection has been considered for long [39-41] and the effect of applying it outside of the cross-validation has been studied previously for more general datasets, but not for radiomics datasets. Refaeilzadeh et al. consider pair-wise comparison of feature selection algorithms in the setup of cross-validation, and argue that especially for small datasets correct application of crossvalidation is wasteful as not all data is used for feature selection, thus inducing another bias. They conducted an experiment on low-dimensional synthetic datasets and showed both methods have different biases, up to $7 \%$, and in the end, they do not differ significantly. Our results do not contradict these findings, since our datasets are high-dimensional, where it is known that the bias can be larger. In the same spirit, Aldehim and Wang considered 10 real-world and 14 synthetic datasets using 4 different feature selection methods and 3 classifiers [42]. They concluded that for datasets with large samples there is "no noticeable difference", but for smaller datasets a bias has occurred". Our experiments confirm this finding, we demonstrated that for radiomics datasets the bias is actually even larger than they have observed, possibly not only because of smaller sample sizes, but also because of highly correlated features.

Analysis of highly correlated features is difficult and can lead to spurious results: Using a cohort of patients with head and neck cancer, Ger et al. demonstrated that tumor volume alone obtained a higher AUC-ROC than a model based on radiomics features, and that the combination of both surprisingly decreased the performance [43]. In the same spirit, Welch et al. showed that three out of four features of the seminal radiomics model presented by Aerts et al. [1] highly correlated with tumor volume and that tumor volume alone yields the same performance, basically questioning whether radiomics beyond tumor volume has any benefit at all in this case [44].

Our focus was to show that an incorrect feature selection indeed leads to a large positive bias when compared to the correct application of feature selection. However, some limitations apply to our study. Foremost, without explicit independent validation sets, the true extent of the bias cannot be determined. A reasonable solution would have been to either split these off from the datasets or to use validation sets where given. Since such validation sets were not always available, and because of the low sample sizes of the datasets, we were unable to do this.

Regarding the experiments, several choices had to be made. We tried to use more common feature selection and classifiers but only tuned a few of the multitude of hyper-parameters because of computational restrictions. It can be expected that better tuning will lead to even higher bias.

Technically, the normalization and also the imputation of missing values must also be part of the crossvalidation. In this study, we forfeit this and applied the normalization and imputation as preprocessing steps to avoid another source of bias, since both do not use the outcome, and thus the influence of them should be rather small when compared to the feature selection. For the same reason, other techniques which are often used in radiomics studies, like outlier removal, or synthetically generating additional training samples to overcome imbalanced problems were not applied. Therefore, our results can be understood as a lower estimate to the bias.

There are still open questions, for example, we used tenfold cross-validation, and it is not clear how a different validation scheme like a fivefold $\mathrm{CV}$ or leave-oneout CV will impact the observed bias. However, such studies would not change the fact that incorrect application of feature selection and cross-validation is selfevidently wrong, regardless of the bias that could or 


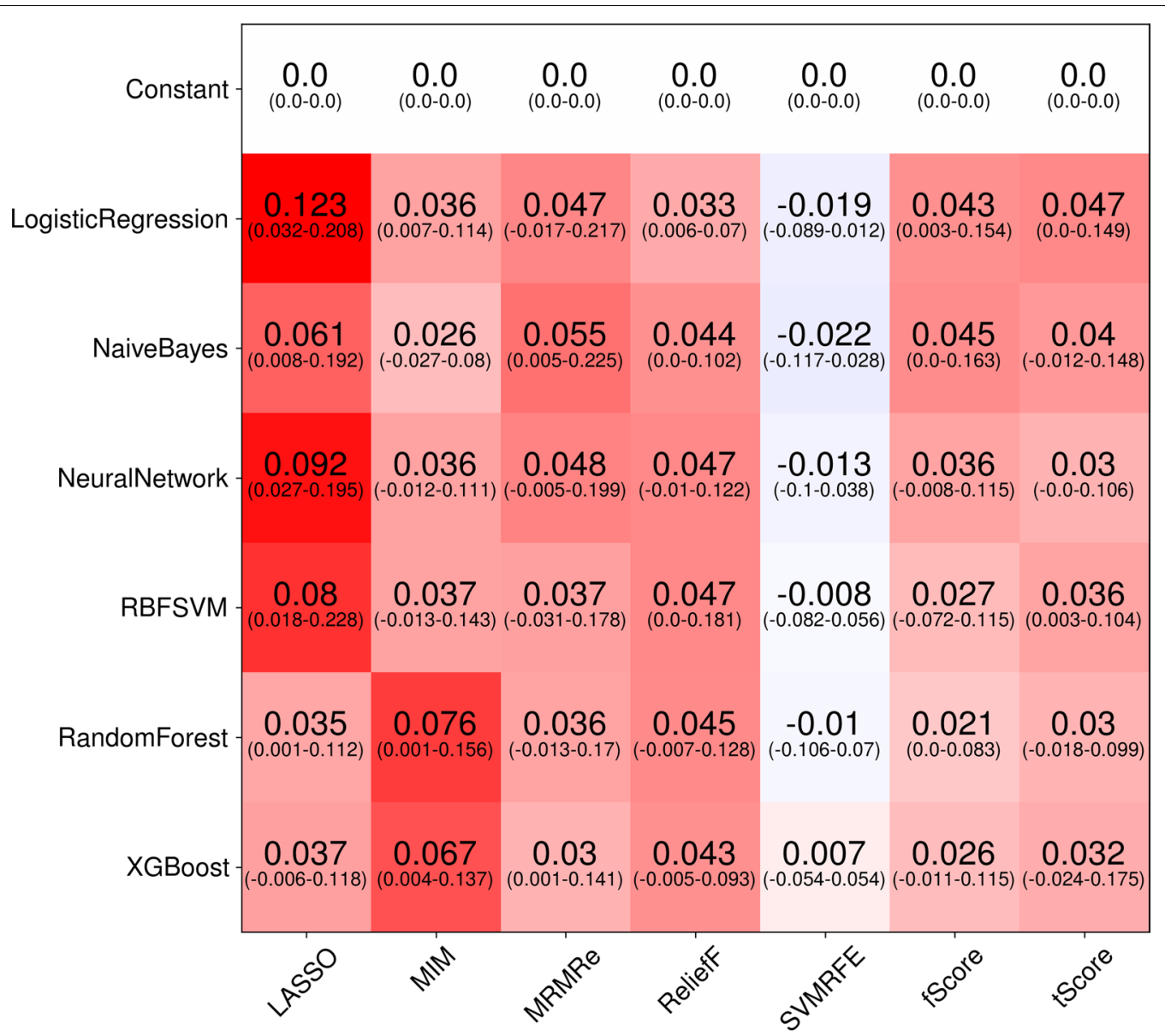

Fig. 4 Mean bias in AUC-ROC for each combination of feature selection and classification method over all datasets. To obtain these, for each of the 10 datasets, the largest difference in AUC-ROC for a given combination between correct and incorrect application of CV is computed, resulting in 10 differences. The mean of these differences is denoted, with the corresponding ranges below in parentheses. Since the displayed mean is an average of all datasets, a conclusion about the bias for a single dataset is not possible. For example, using SVM-RFE with a random forest shows almost no bias in mean (-0.01), but the difference for a single dataset can be as high as 0.07 in AUC-ROC

could not occur. They can only increase the awareness of this problem since this kind of misapplication seems still to be present in recent studies.

\section{Conclusion}

We have shown that incorrectly applying feature selection before cross-validation to high-dimensional radiomics data can lead to positive bias because of data leakage.

\footnotetext{
Abbreviations

AUC: Area under the curve; AUC-F1: Area under the precision-recall curve; AUC-ROC: Area under the curve of receiver-operating characteristics; CT: Computed tomography; CV: Cross-validation; FDG-PET: Fluorodeoxyglucose-positron emission tomography; MIM: Mutual information maximization; MRMRe: Minimum redundancy, maximum relevance ensemble; NN: Neural network; RBF-SVM: Support vector machine with radial basis function kernel (RBF-SVM); RF: Random forests; SVM: Support vector machine; US: Ultrasound.
}

\section{Supplementary Information}

The online version contains supplementary material available at https://doi. org/10.1186/s13244-021-01115-1.

Additional file 1. Details on the datasets and experiments performed.

Additional file 2. Results of all experiments.

\section{Acknowledgements}

AD would like to thank all cited authors who made their datasets publicly available.

\section{Authors' contributions}

$A D$ is the author of this article and conducted the whole study as well as writing the manuscript. The author read and approved the final manuscript.

\section{Funding}

Open Access funding enabled and organized by Projekt DEAL.

\section{Availability of data and materials}

All datasets generated and/or analyzed during the current study are publicly available (https://github.com/aydindemircioglu/radCV). 


\section{Declarations}

\section{Ethics approval and consent to participate}

This study is retrospective in nature and uses only publicly available datasets. The local Ethics Committee (Ethik-Kommission, Medizinische Fakultät der Universität Duisburg-Essen, Germany) waived therefore the need for an ethics approval.

\section{Consent for publication}

Not applicable.

\section{Competing interests}

The authors declare that they have no competing interests.

Received: 15 July 2021 Accepted: 25 October 2021

Published online: 24 November 2021

\section{References}

1. Aerts HJWL, Velazquez ER, Leijenaar RTH et al (2014) Decoding tumour phenotype by noninvasive imaging using a quantitative radiomics approach. Nat Commun 5:1-9. https://doi.org/10.1038/ncomms5006

2. Lambin P, Leijenaar RTH, Deist TM et al (2017) Radiomics: the bridge between medical imaging and personalized medicine. Nat Rev Clin Oncol 14:749-762. https://doi.org/10.1038/nrclinonc.2017.141

3. Keek SA, Leijenaar RT, Jochems A, Woodruff HC (2018) A review on radiomics and the future of theranostics for patient selection in precision medicine. Br J Radiol 91:20170926. https://doi.org/10.1259/bjr.20170926

4. Valdora F, Houssami N, Rossi F, Calabrese M, Tagliafico AS (2018) Rapid review: radiomics and breast cancer. Breast Cancer Res Treat 169:217-229. https://doi.org/10.1007/s10549-018-4675-4

5. Park JE, Kim HS, Kim D et al (2020) A systematic review reporting quality of radiomics research in neuro-oncology: toward clinical utility and quality improvement using high-dimensional imaging features. BMC Cancer 20:29. https://doi.org/10.1186/s12885-019-6504-5

6. Traverso A, Wee L, Dekker A, Gillies R (2018) Repeatability and reproducibility of radiomic features: a systematic review. Int J Radiat Oncol 102:1143-1158. https://doi.org/10.1016/j.jirobp.2018.05.053

7. Baeßler B, Weiss K, Pinto dos Santos D (2019) Robustness and reproducibility of radiomics in magnetic resonance imaging: a phantom study. Invest Radiol 54:221-228. https://doi.org/10.1097/RLI.0000000000000530

8. Bernatz S, Zhdanovich Y, Ackermann J et al (2021) Impact of rescanning and repositioning on radiomic features employing a multi-object phantom in magnetic resonance imaging. Sci Rep 11:14248. https://doi.org/ 10.1038/s41598-021-93756-x

9. Pinto dos Santos D, Dietzel M, Baessler B (2021) A decade of radiomics research: are images really data or just patterns in the noise? Eur Radiol 31:1-4. https://doi.org/10.1007/s00330-020-07108-w

10. Kuncheva LI, Matthews CE, Arnaiz-González Á, Rodríguez JJ (2020) Feature Selection from High-Dimensional Data with Very Low Sample Size: A Cautionary Tale. arXiv:2008.12025 Cs Stat

11. Song J, Yin Y, Wang H, Chang Z, Liu Z, Cui L (2020) A review of original articles published in the emerging field of radiomics. Eur J Radiol 127:108991. https://doi.org/10.1016/j.ejrad.2020.108991

12. Fournier $L$, Costaridou $L$, Bidaut $L$ et al (2021) Incorporating radiomics into clinical trials: expert consensus endorsed by the European Society of Radiology on considerations for data-driven compared to biologically driven quantitative biomarkers. Eur Radiol. https://doi.org/10.1007/ s00330-020-07598-8

13. Zwanenburg A, Vallières M, Abdalah MA et al (2020) The image biomarker standardization initiative: standardized quantitative radiomics for highthroughput image-based phenotyping. Radiology 295:328-338. https:// doi.org/10.1148/radiol.2020191145

14. Sanduleanu S, Woodruff HC, de Jong EEC et al (2018) Tracking tumor biology with radiomics: a systematic review utilizing a radiomics quality score. Radiother Oncol 127:349-360. https://doi.org/10.1016/j.radonc. 2018.03.033

15. Park JE, Kim D, Kim HS et al (2020) Quality of science and reporting of radiomics in oncologic studies: room for improvement according to radiomics quality score and TRIPOD statement. Eur Radiol 30:523-536. https://doi.org/10.1007/s00330-019-06360-z

16. Grimm LJ (2021) Radiomics: a primer for breast radiologists. J Breast Imaging 3:276-287. https://doi.org/10.1093/jbi/wbab014

17. Xiong X, Wang J, Hu S, Dai Y, Zhang Y, Hu C (2021) Differentiating between multiple myeloma and metastasis subtypes of lumbar vertebra lesions using machine learning-based radiomics. Front Oncol 11:601699

18. Wen L, Weng S, Yan C et al (2021) A radiomics nomogram for preoperative prediction of early recurrence of small hepatocellular carcinoma after surgical resection or radiofrequency ablation. Front Oncol 11:657039

19. Wang Q, Zhang Y, Zhang E et al (2021) Prediction of the early recurrence in spinal giant cell tumor of bone using radiomics of preoperative CT: Long-term outcome of 62 consecutive patients. J Bone Oncol 27:100354

20. Shi Y, Wahle E, Du Q et al (2021) Associations between statin/omega3 usage and MRI-based radiomics signatures in prostate cancer. Diagnostics 11:85

21. Sartoretti E (2021) Amide proton transfer weighted (APTw) imaging based radiomics allows for the differentiation of gliomas from metastases. Sci Rep 11:1-8

22. Ni XQ, Yin HK, Fan GH, Shi D, Xu L, Jin D (2021) Differentiation of pulmonary sclerosing pneumocytoma from solid malignant pulmonary nodules by radiomic analysis on multiphasic CT. J Appl Clin Med Phys 22:158-164. https://doi.org/10.1002/acm2.13154

23. Naranjo ID, Gibbs P, Reiner JS et al (2021) Radiomics and machine learning with multiparametric breast MRI for improved diagnostic accuracy in breastcancer diagnosis. Diagnostics 11:919

24. Mulford K, Chen C, Dusenbery K et al (2021) A radiomics-based model for predicting local control of resected brain metastases receiving adjuvant SRS. Clin TransI Radiat Oncol 29:27-32

25. Li Z, Ma X, Shen F, Lu H, Xia Y, Lu J (2021) Evaluating treatment response to neoadjuvant chemoradiotherapy in rectal cancer using various MRIbased radiomics models. BMC Med Imaging 21:1-10

26. Krajnc D, Papp L, Nakuz TS et al (2021) Breast tumor characterization using [18F]FDG-PET/CT imaging combined with data preprocessing and radiomics. Cancers 13:1249

27. Kihira S, Tsankova NM, Bauer A et al (2021) Multiparametric MRI texture analysis in prediction of glioma biomarker status: added value of MR diffusion. Neuro-Oncol Adv 3:vdab051. https://doi.org/10.1093/noajnl/ vdab051

28. Kawahara D, Tang $X$, Lee CK, Nagata $Y$, Watanabe $Y$ (2021) Predicting the local response of metastatic brain tumor to gamma knife radiosurgery by radiomics with a machine learning method. Front Oncol 10:569461

29. Bevilacqua A, Mottola M, Ferroni F, Rossi A, Gavelli G, Barone D (2021) The primacy of high B-value 3T-DWI radiomics in the prediction of clinically significant prostate cancer. Diagnostics 11:739

30. Carvalho S, Leijenaar RTH, Troost EGC et al (2018) 18F-fluorodeoxyglucose positron-emission tomography (FDG-PET)-Radiomics of metastatic lymph nodes and primary tumor in non-small cell lung cancer (NSCLC): a prospective externally validated study. PLoS One 13:e0192859. https:// doi.org/10.1371/journal.pone.0192859

31. Hosny A, Parmar C, Coroller TP et al (2018) Deep learning for lung cancer prognostication: a retrospective multi-cohort radiomics study. PLOS Med 15:e1002711. https://doi.org/10.1371/journal.pmed.1002711

32. Ramella S, Fiore M, Greco C et al (2018) A radiomic approach for adaptive radiotherapy in non-small cell lung cancer patients. PLoS One 13:e0207455. https://doi.org/10.1371/journal.pone.0207455

33. Toivonen J, Montoya Perez I, Movahedi P et al (2019) Radiomics and machine learning of multisequence multiparametric prostate MRI: towards improved non-invasive prostate cancer characterization. PLoS One 14:e0217702. https://doi.org/10.1371/journal.pone.0217702

34. Keek S, Sanduleanu S, Wesseling F et al (2020) Computed tomographyderived radiomic signature of head and neck squamous cell carcinoma (peri)tumoral tissue for the prediction of locoregional recurrence and distant metastasis after concurrent chemo-radiotherapy. PLoS One 15:e0232639. https://doi.org/10.1371/journal.pone.0232639

35. Li J, Liu S, Qin Y, Zhang Y, Wang N, Liu H (2020) High-order radiomics features based on T2 FLAIR MRI predict multiple glioma immunohistochemical features: a more precise and personalized gliomas management. PLoS One 15:e0227703. https://doi.org/10.1371/journal.pone.0227703

36. Park VY, Han K, Kim HJ et al (2020) Radiomics signature for prediction of lateral lymph node metastasis in conventional papillary thyroid 
carcinoma. PLoS One 15:e0227315. https://doi.org/10.1371/journal.pone. 0227315

37. Song Y, Zhang J, Zhang Y et al (2020) FeAture Explorer (FAE): a tool for developing and comparing radiomics models. PLoS One 15:e0237587. https://doi.org/10.1371/journal.pone.0237587

38. Parmar C, Grossmann P, Bussink J, Lambin P, Aerts HJ (2015) Machine learning methods for quantitative radiomic biomarkers. Sci Rep 5:1-11. https://doi.org/10.1038/srep13087

39. Guyon I, Elisseeff A (2003) An introduction to variable and feature selection. J Mach Learn Res 3:1157-1182

40. Dash M, Liu H (1997) Feature selection for classification. Intell Data Anal 1:131-156. https://doi.org/10.1016/S1088-467X(97)00008-5

41. Chandrashekar G, Sahin F (2014) A survey on feature selection methods. Comput Electr Eng 40:16-28. https://doi.org/10.1016/j.compeleceng. 2013.11.024
42. Aldehim G, Wang W (2017) Determining appropriate approaches for using data in feature selection. Int J Mach Learn Cybern 8:915-928. https://doi.org/10.1007/s13042-015-0469-8

43. Ger RB, Zhou S, Elgohari B et al (2019) Radiomics features of the primary tumor fail to improve prediction of overall survival in large cohorts of CTand PET-imaged head and neck cancer patients. PLoS One 14:e0222509. https://doi.org/10.1371/journal.pone.0222509

44. Welch ML, McIntosh C, Haibe-Kains B et al (2019) Vulnerabilities of radiomic signature development: the need for safeguards. Radiother Oncol 130:2-9. https://doi.org/10.1016/j.radonc.2018.10.027

\section{Publisher's Note}

Springer Nature remains neutral with regard to jurisdictional claims in published maps and institutional affiliations.

\section{Submit your manuscript to a SpringerOpen ${ }^{\circ}$ journal and benefit from:}

- Convenient online submission

- Rigorous peer review

- Open access: articles freely available online

- High visibility within the field

- Retaining the copyright to your article

Submit your next manuscript at $\boldsymbol{\text { springeropen.com }}$ 